\title{
Whole Body Mechanical Vibrations of Passengers Travelling in Busses in Sri Lanka
}

\author{
R. Wijesiriwardana, M. Vignarajah, P.Kathirgamanathan, T.U. Gnanasena \& D.M.P.C. Dissanayake
}

\begin{abstract}
One of the main passenger transportations in Sri Lanka is the bus. Most of these busses used for the passenger transportation do not have proper vibration dampers and passengers who travel in them are subjected to severe vibration levels as most of the time they are travelling standing up rather than sitting down on comfortable seats. Sever vibration levels produced by the engines of the busses and the poor road conditions in Sri Lanka are directly transfer to the passengers due to poor vibration damping of the busses. This paper discusses the vibration levels that the passengers travelling in Sri Lankan busses encounter by using accelerometers and the mechanical modelling of the passenger whole body vibrations. A new model is put forward to account for the nonlinear effects. Also the paper compares the passenger vibration levels with the international standards for safer passenger vibration levels (ISO2631). First part of the paper discusses the power spectral densities of the vibrations generated in the busses and also transferring from the road on to different locations of the busses where the passengers are normally travelling. Also the paper further discusses improved lumped parameter based mechanical dynamics model for standing up travelers subjected these vibrations, catering for the nonlinear effects by using additional viscous springs and dampers. Final part of the paper discusses the effectiveness of the use of commonly available elastic soles, elastic insert pads or elastic slippers foot ware to reduce the harmful vibrational effects of frequent travelers.
\end{abstract}

Keywords —whole body vibration, passenger bus, ISO2631

\section{Introduction}

There are many public transport service modes in Sri Lanka. Busses, trains, cars, motor bikes, bicycles, motor bikes and three wheelers are some of the commonly used passenger travelling modes in Sri Lanka[1,2]. Out of them busses are the most commonly used transport mode in Sri Lanka [1,2]. Figure 1.0a shows a typical Sri Lankan bus. The bus mode of transportation is popular due to their availability, and economical ticket prices. However their construction is very robust and uncomfortable. These busses consist of lorry engines and chassis covered by a metal frame with seats without proper dampers or vibration absorbers for the dynamic variations. Figure 1.0b shows that only leaf spring dampers are available for damping the road vibrations from the tires. In addition, they are powered with very high power diesel engines that produce wider frequency bandwidth of high intense vibrations which are not also properly damped.

\footnotetext{
R. Wijesiriwardana

M. Vignarajah

P.Kathirgamanathan

T.U. Gnanasena

D.M.P.C. Dissanayake

University of Jaffna

Sri Lanka
}

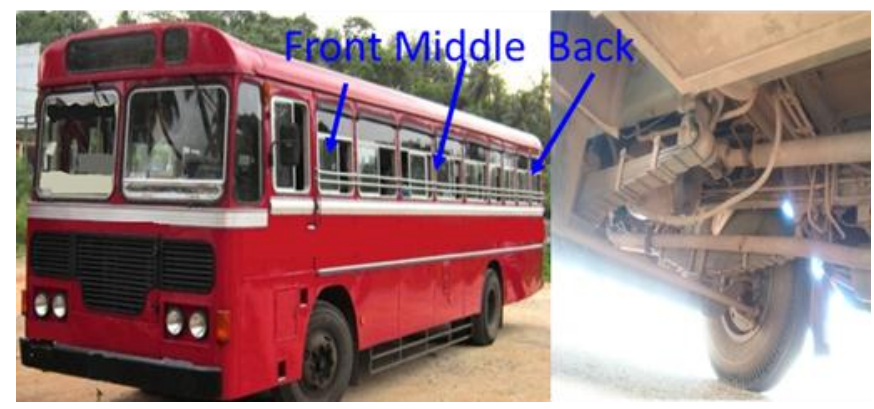

Figure 1.0a Bus in Sri Lanka

Figure 1.0b Under view chassis

Due to the poor maintenance most of these busses engines are in poor operational conditions emitting unburnt diesel and carbon particles into the atmosphere together with acoustic noise pollution. Moreover due to the uneven roads and the congested traffic conditions in Sri Lanka the passengers have to bare additional burden of vibrations and spent additional time on such a hostile environment during their daily commutes.

Usually school children, working class people and elderly people who are travelling in these busses undergoes very harsh conditions with respect to mechanical whole body vibrations during their daily commutes. On average regular passenger stay inside these busses under harsh conditions for 1 hour and 45minutes in their daily commutes [1]. Even though for non-regular passengers 1 hour and 45 minutes of undergoing these vibrations are tolerable as they can rest and recover, but for daily frequent travelers especially for the school children and the elderly these conditions in addition to the short term uncomfortability, could contribute to long term health issues[3]. In addition bus drivers and the conductors are working 12 to 16 hours minimum shift per day I Sri Lanka and they are also vulnerable to have a higher dosage of vibrational energy exposures.

According to the international passenger and vibration standards [3], the maximum amount of allowable vibrations levels that the human body can tolerate depends on the location, direction of the vibration, frequency, age, duration of the vibration, posture mode (sitting or standing) and also the rest duration between vibration levels. Also according to ISO2631-1, ISO2631-4, ISO2631-5 frequencies from $0.1 \mathrm{~Hz}$ to $0.5 \mathrm{~Hz}$ results in motion sickness and Frequencies from 0.5 to $80 \mathrm{~Hz}$ are transmitted from seat to the body of the seated. Higher Frequency health effects from $80 \mathrm{~Hz}$ to $1 \mathrm{kHz}$ are not known due to non-availability. In addition these standards do not specify the health effects of the standing up or recumbent positions due to non-availability of data. In Sri Lanka most of the passengers are travelling standing up during the rush hours. Moreover under seated conditions according to the these standards long term high intensity whole body vibrations indicates an increased health risk to lumbar spine and the connected nervous system of the segments affected. This may trigger by the not only the 
vertical accelerations but also the horizontal and torsional components. Also contribute strongly to the degenerative processes in the lumbar segments such as spondylosys deformance, osteochondrosis intervertebralis and arthrosis deformance[3].Not only the whole body vibration effects the bones and the joints they also assumed to be effecting the digestive system, the genitals-urinary system and the female reproductive systems. In general according to the standard it takes several years for health changes caused by the whole body vibration to occur and therefore it is important not to be exposed for high intense whole body vibrations regularly for long durations of time. Moreover the stands specifies the discomfort levels at sitting conditions for different acceleration levels where great than $2 \mathrm{~ms}^{-2}$ is consider to be extremely uncomfortable however this may be different for Sri Lankan bus passengers. Even though according to the ISO2631-1, Figure 2.0 shows the maximum vibration tolerable levels and caution zones for a given exposure duration. The detailed health effects are not known for lover vibration levels as clear documented data are not available. Above the caution zone the health risks are very likely to happen. Average person daily vibration exposures are measured by using either vibration exposure dosage value (VDV) given in equation (1).

$$
a_{w} T^{\frac{1}{2}} \text { or } a_{w} T^{\frac{1}{4}}
$$

Where $a_{w}$ is the resultant weighted acceleration and $T$ is the time of exposure.

However, the data of these standards were taken with the Caucasians who are stronger built and hence more vibration tolerable than the South Asians such as Sri Lankans. In Sri Lanka such standards have not been developed so far. Depending on the body structure, nutrition levels, bone constituents and bone structure, regular South Asian the maximum tolerable values may be lower than the values given in the international standards [3]. In addition, these standards clearly indicate proper resting is required for the body and the bones to recover and remodeled themselves after undergoing severe whole body vibration exposure. Also if the proper resting or remodeling of the bones does not happen, it could leads to aggravation and propagation of the micro cracks and could even results in bone fatigue (depend on the strain and strain frequencies) and cartilage damages $[3,4,5]$. In addition to the health effects other adverse side effects results in exposure to a high dosage of whole body vibrations are decreased performance levels, temporary threshold shifts, reduce motion control, impaired vision, discomfort and fatigue decreased job proficiency.

Usually passengers travel either sitting or standing up and during this research we have measured the vibrations of the passengers at three locations front, middle and back while sitting and standing up at each location shown in Figure 1.0). In addition, the standing and sitting passengers were asked to wear either normal shoes or elastic slippers and the vibrations were measured under each condition and compared with the standards [3] permissible chart at Figure 2.0. Most of the research work on passenger whole body vibrations have been done with trains, tractors, metros, cars, coaches[8,9,10,11,12], and some simulation work was carried out under sitting conditions[24]. Our paper discusses the whole body vibration of passengers travelling in Sri Lankan public busses under standing and sitting conditions, with and without wearing commonly used rubber slippers.

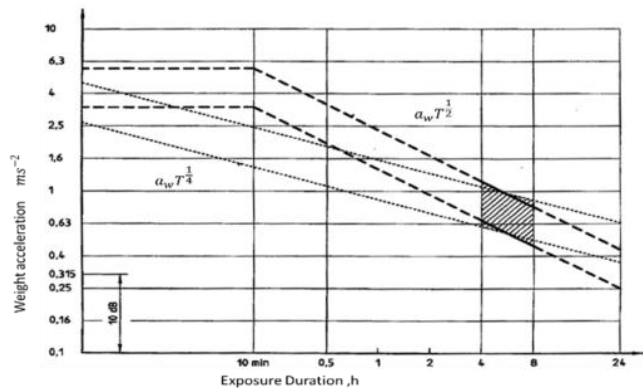

Figure 2.0 Exposure duration vs maximum allowable accelerations and caution region (from ISO2631-1)

\section{Mechanical Vibrations of Buses}

The diesel engines of the busses produce large amplitudes of vibrations over wider frequency band width. In addition, under poor road conditions the vibration spectrum gets modulated with the road conditions induced vibrations and due to the traffic conditions on the roads sudden jerks produce additional power spectral components into this vibration spectrum. Most busses have the leaf spring type shock absorbers but not liquid damping that can absorb the jerks and the speed variations. Also the tires do not damp out the road vibrations and neither transfer most of the engine vibrations on to the road. The vibrational power spectrum from the engine to the body of the bus depends on the RPM of the engine and the coupling between the engine and the transmission and the engine and the body. The vibrations generated between the road and the bus depend on the road conditions and the velocity, acceleration, the jerk of the bus and the coupling between the road and the tires.

\section{Mechanical Vibrations Measurement Method}

We have used accelerometers connected at front, middle and the back to the floor as shown in the Figure 3.0 to measure the floor vibrations. The acceleration data is logged in quasi real time by using a tri-axial accelerometers DAQ and computer (Figure 4.0). We have put tri-axial accelerometers on the floor, shank and thigh of a person as shown in the Figure 3.0 to measure the transfer of vibrational energy from the floor to the shank and then to the thigh and the above segments. The measurements were taken at three locations front, middle and back of the bus while standing up and, seated with shoes and with elastic slippers respectively. In addition, three different body shapes of young adults were selected with three different body mass indexes (BMI) 18, 25 and 30 respectively for the experiment. The captured quasi real time data are then converted into frequency domain by using Discrete Fourier Transformation (DFT) then the power spectrum of the accelerations were obtained. The data is taken from three busses travelling on the same terrain and the average power spectrum was obtained. The Figure 5.0 shows the typical seating arrangement of the driver. Most of the mechanical vibrational effects transferred on to the structure of the bus, then on to the passengers who are travelling inside and also into the surrounding air. 
Proc. of the Eighth International Conference On Advances in Mechanical, Aeronautical and Production Techniques - MAPT 2018 Copyright (C) Institute of Research Engineers and Doctors. All rights reserved. ISBN: 978-1-63248-147-4 doi: 10.15224/ 978-1-63248-147-4-20

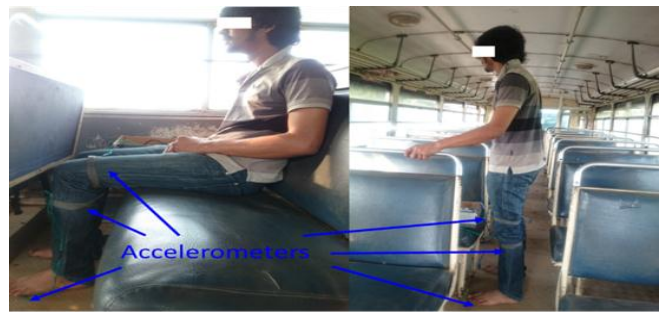

Figure 3.0 Placement of accelerometers under sitting and standing orientations of the passenger

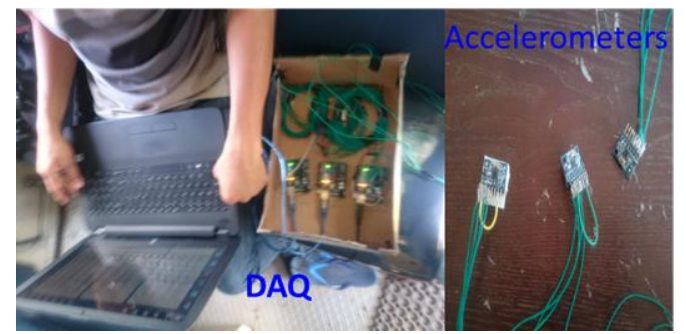

Figure 4.0 Accelerometers and the DAQ

\section{Transmission and Absorption of the Mechanical Vibrations by Human Tissues}

During this study except the vibrations from the bus, other self-generated movements of the passengers are not considered. The acceleration readings were taken by using tri-axial accelerometers when a passenger was sitting down or standing up as shown in the Figure 3.0. When the vibrations occur on the floor of the buss the vibrational energy get transferred into the tissues of the passengers and some of them get absorbed into the tissues and the rest makes the tissue vibrations[6]. The part absorbed into the tissue results developing and aggravating micro cracks in the bones $[3,4,5]$,tissue plastic deformations and heat due to friction. Proper resting is required to get back to the original stage of the tissue $[3,4,5]$. The permissible level of exposure depend upon the frequency values and the Figure 6.0 shows the vertical permissible exposure durations with different frequencies of exposure. Negligence of these levels of the whole body vibrations could results in fatigue decreased job proficiency. However with this respect standing passengers context, data are not available. Human tissue orientations are complex nonlinear continuum materials arrangements with anisotropic properties. different tissue types such as skin, muscles, tendons, ligaments, fats and bones connects interacts to make dynamic mechanical system.

\section{Lump Parameters based Mechanical Dynamics Modelling of Human Body}

Two mathematical models were used to analyses the dynamics of the whole body vibrations of passengers understanding and sitting conditions. We have used linear masses, springs and dampers model to model the vibration transfer from the floor to shank then to the thigh of the standing up and sitting down passengers. Similar to mass spring model put forward by Rasmussen et al[7] shown in
Figure 7.0. We also introduced additional stiffness coefficient and a viscous damping coefficient between the Thigh and above segments and the foot in order to absorb the nonlinear effects (Figure 9.0). We have adopted Figur 9.0 model to analyze the vibration transmission that depends on the Frequency [6] sown in Figure 8.0a and Figure 8.0b. It is understood that human body parts and tissues have different vibrational frequencies and as the vibrations comes into the body the transmissibility depend on the frequency (Figure 8.0). Figure 8.0a shows transmissibility of vertical vibration from table to various parts of the body of a standing human subject as a function of frequency (from Mechanical Vibration and Shock Measurements by Jens Trampe Broch ISBN 878735534 5). Figure 8.0b shows transmissibility of vertical vibration from table to various parts of a seated human subject as a function of frequency (from Mechanical Vibration and Shock Measurements by Jens Trampe Broch ISBN 878735534 5). We have used the following equation (2) in matrix form for the simulations and estimations of the forces.

$$
[M]\{X\}+[C]\{X\}+[K]\{X\}=\{F\}
$$

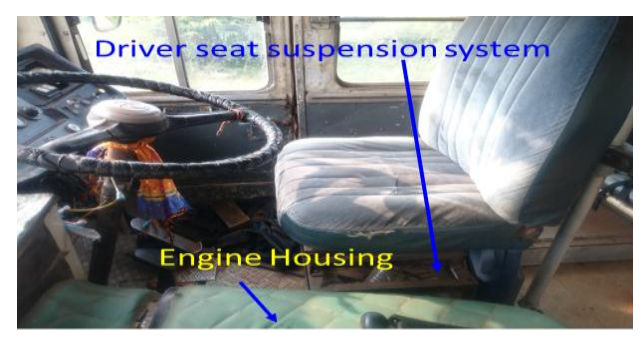

Figure 5.0 Seating arrangement of the driver

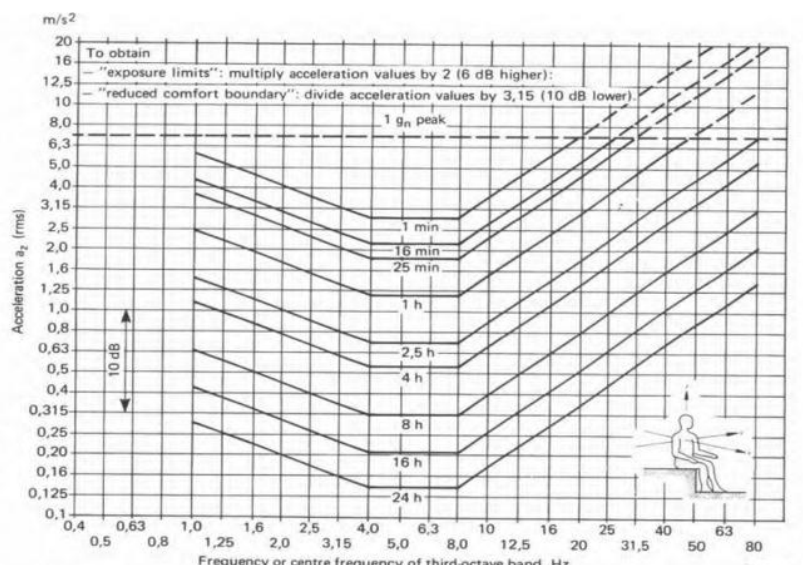

Figure 6.0 Vertical vibration exposure criteria curves defining equal fatigue-decreased proficiency boundaries (from Mechanical Vibration and Shock Measurements by Jens Trampe Broch ISBN 878735534 5)

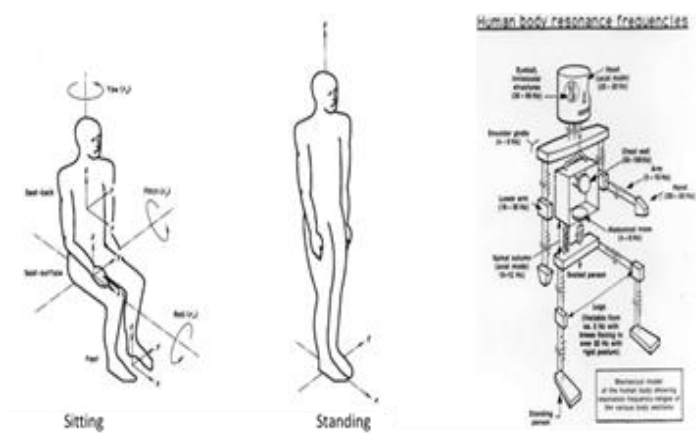

Figure 7.0 Mass Spring Damper model for standing and sitting down passenger model (form Rasmussen et. al, [7]). 
Proc. of the Eighth International Conference On Advances in Mechanical, Aeronautical and Production Techniques - MAPT 2018 Copyright (C) Institute of Research Engineers and Doctors. All rights reserved. ISBN: 978-1-63248-147-4 doi: 10.15224/ 978-1-63248-147-4-20
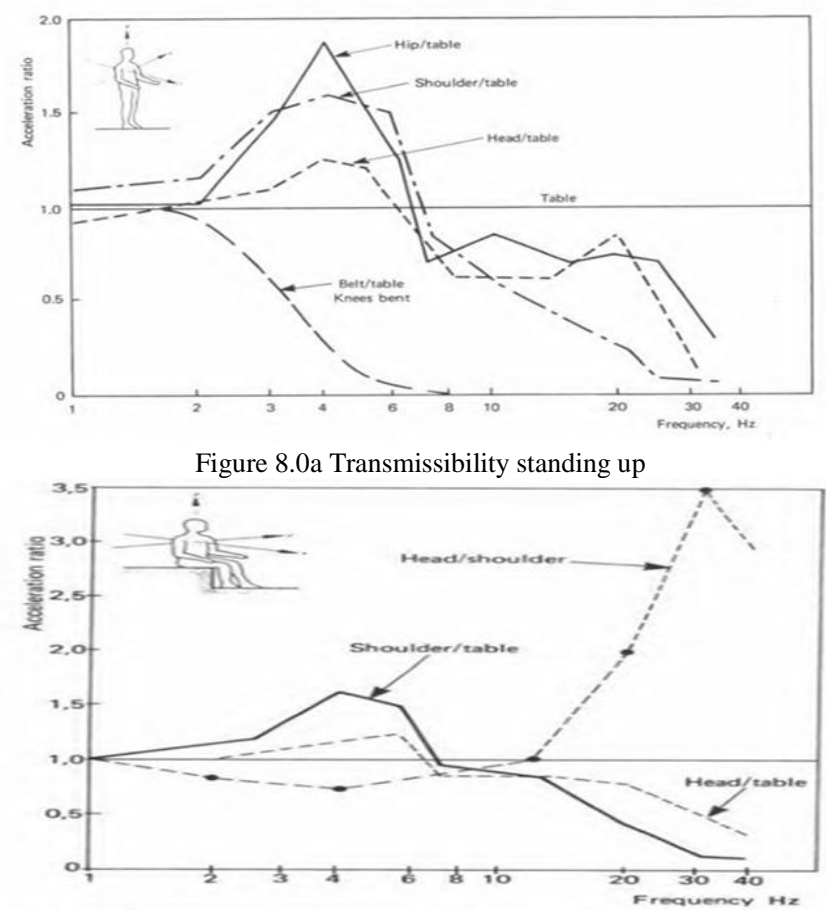

Figure $8.0 \mathrm{~b}$ Transmissibility sitting up

Where $[M]$ is the mass matrix,$\{X\}$ is the displacement vector, $\{X\}$ is the velocity vector, $\{X\}$ is the acceleration vector, $[C]$ is the damping matrix and $[\mathrm{K}]$ is the stiffness matrix and $\{\mathrm{F}\}$ is the residual force. The system of equations can be solved in either time domain or the frequency domain. Since $\{F\}$ is not known it can be find by using inverse methods using the $\{\mathcal{X}\}$ information. Hence the $\{F\}$ power spectral density can be obtained. Since three locations readings of accelerations were measured to find $\{F\}$ the model was reduced into 3 acceleration components taking from the thigh and above as a single mass shown in the Figure 9.0. The velocity $\{\boldsymbol{X}\}$ and the displacement $\{X\}$ found by using average acceleration method of integration [13]. The thigh and above sections mass effects are lumped to a single mass $M_{3}$. Shank mass and the foot mass are taken as $M_{2}$ and $M_{1}$.The linear viscous damping coefficient between $\mathrm{i}^{\text {th }}, \mathrm{j}^{\text {th }}$ masses is $C_{\text {if }}$ and the linear stiffness coefficient between $\mathrm{i}^{\text {th }}$, $\mathrm{j}^{\text {th }}$ masses is $K_{\text {ij }}$. Then viscous damping coefficient matrix [C], stiffness coefficients matrix $[\mathrm{K}]$ and the mass matrix $[\mathrm{M}]$ are given by the equation $3 \mathrm{a}, 3 \mathrm{~b}$ and $3 c$ respectively. Then the resultant force on the $i^{\text {th }}$ mass is $F_{i}$ can be estimated with equation (4a). Also the total force $(\mathrm{F})$ exerted on the body at the foot by the floor is estimated by using the equation (2).

$$
\begin{aligned}
& {[C]=\left[\begin{array}{ccc}
C_{1 a}+C_{12} & -C_{12} & -C_{1 a} \\
-C_{12} & C_{12}+C_{2 a} & -C_{2 a} \\
-C_{1 a} & -C_{2 a} & C_{1 a}+C_{2 a}
\end{array}\right]} \\
& {[K]=\left[\begin{array}{ccc}
K_{1 a}+K_{12} & -K_{12} & -K_{1 a} \\
-K_{12} & K_{12}+K_{2 a} & -K_{2 a} \\
-K_{1 a} & -K_{2 a} & K_{1 a}+K_{2 a}
\end{array}\right]} \\
& {[M]=\left[\begin{array}{ccc}
M_{1} & 0 & 0 \\
0 & M_{2} & 0 \\
0 & 0 & M_{a}
\end{array}\right]}
\end{aligned}
$$

the values of the $[\mathrm{C}],[\mathrm{K}]$ matrices were taken by considering the proportion masses and the body mass index(BMI) of the test subjects $[21,22,23]$. The mass matrix $[\mathrm{M}]$ is obtained by measuring the mass of the test subjects and obtaining the proportionate mass of the foot, shank and thigh and above sections [21,22,23]. The mass spring model is improved using additional stiffness coefficient $K_{13}$ and viscous damping coefficient $C_{13}$ between masses $M_{1}$ and $M_{3}$ to overcome the nonlinear effects.

$F_{i}=M_{i} \ddot{X}_{i}$

The estimated forces at the knee $(\mathrm{Fk})$ and the ankle $(\mathrm{Fa})$ are given by equations ( $4 \mathrm{~b})$ and $(4 \mathrm{c})$.

$F_{\mathrm{k}}=K_{23}\left(X_{2}-X_{3}\right)+C_{23}\left(\dot{X}_{2}-\dot{X}_{3}\right)$

$F_{a}=K_{12}\left(X_{1}-X_{2}\right)+C_{12}\left(\dot{X}_{1}-\dot{X}_{2}\right)$

The resultant weighted average acceleration $a_{w}$ is estimated according to the ISO2631-1 standard by using the equation (6).

$$
a_{W F}=\left[\Sigma_{i}\left(W_{i} a_{i}\right)^{2}\right]^{\frac{1}{2}}
$$

Where $a_{w F}$ acceleration in $\mathrm{r}$ direction, $W_{i}$ weighting factor of the $\mathrm{i}^{\text {th }} 3^{\text {rd }}$ octave octave band from table 3 and table 4 of the ISO2631-1 standard, $a_{i}$ is the r.m.s. acceleration of the $i^{\text {th }} 3^{\text {rd }}$ octave band (according to the standard). The resultant r.m.s. acceleration from all three $\mathrm{x}, \mathrm{y}, \mathrm{z}$ directions given by,

$$
a_{w}=\left[k_{x}^{2} a_{w x}^{2}+k_{y}^{2} a_{w y}^{2}+k_{z}^{2} a_{w z}^{2}\right]^{\frac{1}{2}}
$$

Where $k_{x y} k_{y}$ and $k_{z}$ are multiplying factors.

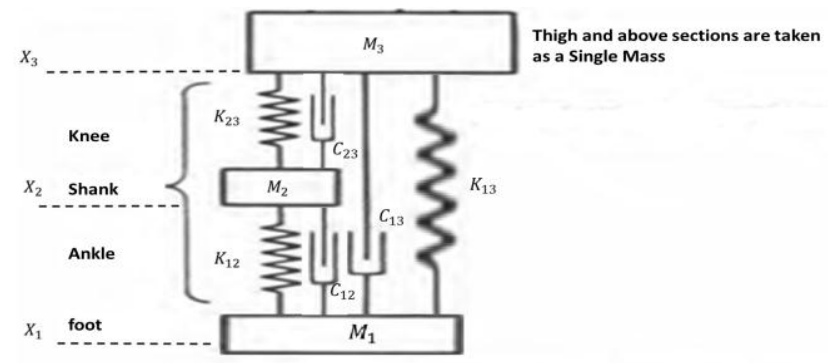

Figure 9.0 Simplified lump parameters model.

\section{Results and Measurements}

Figure 10.0 a,b and $\mathrm{c}$ shows the averaged (all three busses) acceleration with respect to time and normalized acceleration power spectrums on the floor of the buses in the vertical direction at front, middle and back locations.

\begin{tabular}{|l|r|l|l|}
\hline \multicolumn{4}{|c|}{ Sitting } \\
\hline Location & $m s^{-2}$ & $a_{w} T^{\frac{1}{2}}$ & $a_{w} T^{\frac{1}{4}}$ \\
\hline Front & 3.614 & 4.7805 & 4.1567 \\
\hline Middle & 2.252 & 2.9791 & 2.5902 \\
\hline Back & 3.4345 & 4.5434 & 3.9502 \\
\hline
\end{tabular}

Table 1.0a whole body vibrational accelerations and exposure dosages (VDV) sitting without slippers

\begin{tabular}{|l|c|c|r|}
\hline \multicolumn{4}{|c|}{ Standing up } \\
\hline Location & $m s^{-2}$ & $a_{w} T^{\frac{1}{2}}$ & $a_{w} T^{\frac{1}{4}}$ \\
\hline Front & 4.814 & 6.3683 & 5.5369 \\
\hline Middle & 3.9081 & 5.1699 & 4.495 \\
\hline Back & 4.5851 & 5.9332 & 5.1586 \\
\hline
\end{tabular}

Table $1.0 \mathrm{~b}$ whole body vibrational accelerations and exposure dosages (VDV) standing up without slippers 
Proc. of the Eighth International Conference On Advances in Mechanical, Aeronautical and Production Techniques - MAPT 2018 Copyright (C) Institute of Research Engineers and Doctors. All rights reserved. ISBN: 978-1-63248-147-4 doi: 10.15224/ 978-1-63248-147-4-20

\begin{tabular}{|l|r|r|r|}
\hline \multicolumn{4}{|c|}{ Sitting } \\
\hline Location & \multicolumn{1}{|c|}{$m s^{-2}$} & $a_{w} T^{\frac{1}{2}}$ & $a_{w} T^{\frac{1}{4}}$ \\
\hline Front & 2.185 & 2.8905 & 2.5131 \\
\hline Middle & 1.5525 & 2.0538 & 1.7856 \\
\hline Back & 2.0762 & 2.7466 & 2.388 \\
\hline
\end{tabular}

Table 2.0a whole body vibrational accelerations and exposure dosages (VDV) sitting with slippers

\begin{tabular}{|l|r|r|l|}
\hline \multicolumn{4}{|c|}{ Standing up } \\
\hline Location & $m s^{-2}$ & $a_{w} T^{\frac{1}{2}}$ & $a_{w^{\frac{1}{4}}}$ \\
\hline Front & 2.7652 & 3.658 & 3.1804 \\
\hline Middle & 1.8593 & 2.4596 & 2.1385 \\
\hline Back & 2.4712 & 3.2691 & 2.8423 \\
\hline
\end{tabular}

Table $2.0 \mathrm{~b}$ whole body vibrational accelerations and exposure dosages (VDV) standing up with slippers
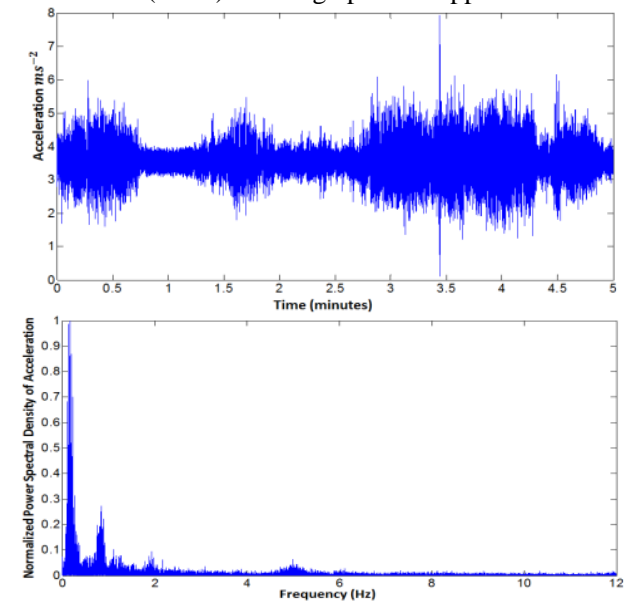

Figure 10.0a Front location vertical acceleration of the bus floor
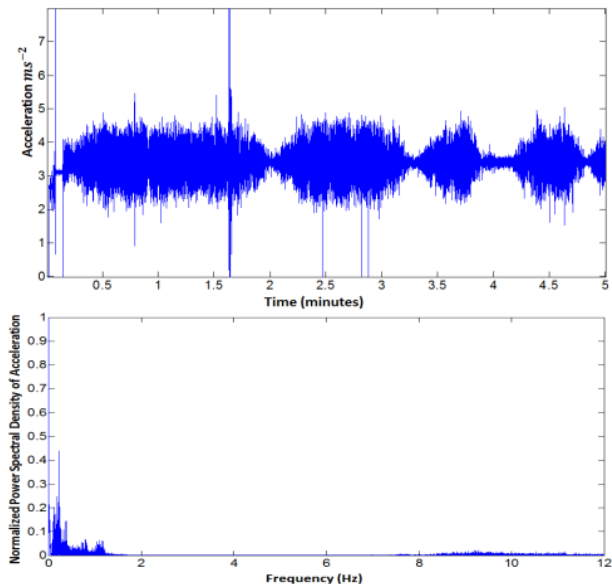

Figure 10.0b Middle location vertical acceleration of the bus floor
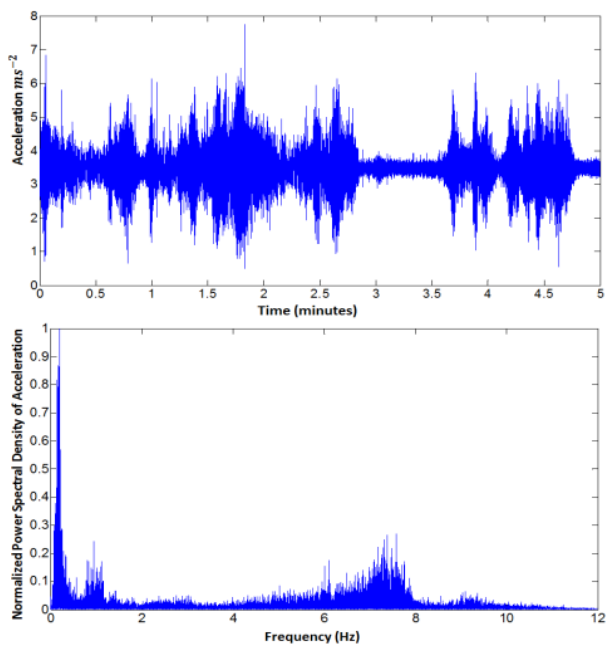

Figure 10.0c Back location vertical acceleration of the bus floor
The calculated resultant r.m.s. values of the whole body vibrational accelerations $\left(a_{w}\right)$ of a passenger at front, middle and back locations and the average vibration dose exposures or the vibration dosage values (VDV) are given in Table $1.0 \mathrm{a}$ under sitting and Table 1.0b under standing up conditions without elastic slippers. Similarly the whole body vibrational accelerations $\left(a_{w}\right)$ of a passenger at front, middle and back locations and the average vibration dose exposure are given in Table 2.0a under sitting and Table $2.0 \mathrm{~b}$ under standing up conditions with elastic slippers. The exposure time is taken as 1.75 hours for an average passenger.

\section{Discussion and Conclusion}

Even though ISO 2631 standards are good starting point much more comprehensive study needed to be carried out in order to understand the effects on health and other functions of the human body due to the whole body vibrations. In addition, this standard also does not provide any information on the whole body vibration effects on different age groups and gender differences as well.

We have observed exposure dosages (VDV) measurements above permissible levels of fatigue-decreased proficiency and also whole body acceleration values inside caution range and above the permissible levels for an average passenger travelling under sitting conditions in most commonly used Sri Lankan passenger busses. In addition we observed that the passengers who are travelling front and back locations of the buses are vulnerable to higher exposure dosages(VDVs) and whole body accelerations than the middle sections inside the busses. We have also observed that passengers who are travelling under standing conditions subjected to more severer conditions, irrespective of the inside location of the busses where the passenger is located at.

The effects of whole body vibration on bones $[4,16,17,18,19,20]$ are studied by various researchers and according these research the whole body vibrations results micro crack of the bones aggravation results in longer bone reformations, bone fatigue and long term bone health problems. Also research showed that long term exposure to whole body vibrations could results people with arthritis and other bone health issues to aggravate. Our results in tables 1 and 2 show that the whole body vibration of the passengers of the busses under standing conditions under goes sever exposure to harmful forces at their ankles, knees and the hips and for sitting passengers the pelvis, the spinal cord and the hip joint undergoes severe exposure to the harmful vibrational forces. Therefore, long term exposure can be really harmful for the bones of the regular passengers. We also observed that the estimated forces were reduced when wearing elastic slipper (compare table 1 and table 2). In addition, the whole body vibration effects the nervous system of the passengers. Studies have been conducted to find out the effects of the nerve conduction velocity and the whole body vibration induced neuropathy $[14,15]$. Our initial study showed that proper vibration reduction is needed inside Sri Lankan busses. In the meantime it would be a better choice to wear slippers, rubber shoe inserts and rubber pads for the frequent 
Proc. of the Eighth International Conference On Advances in Mechanical, Aeronautical and Production Techniques - MAPT 2018

Copyright (C) Institute of Research Engineers and Doctors. All rights reserved. ISBN: 978-1-63248-147-4 doi: 10.15224/ 978-1-63248-147-4-20

travelers, bus drivers and the conductors. Even though we observed a significant reduction in the whole body vibrations and the VDVs when wearing elastic slippers from the data gathered during this research. However the elastic slippers that we used could not bring the whole body vibrations down to the safer levels according to the ISO2631-1 standard. In addition it would be better to use properly designed rubber floor mats on the floor of the buses and improve the seat cushioning. Also more research work needed to be carried out on whole body vibration and the Standard ISO2631 needs improve.

Our mechanical model is an approximation and in future we intended to develop better simulation models for standing passengers by using nonlinear energy based finite element dynamics methods.

\section{Acknowledgment}

We take this opportunity to give our heartiest thanks to the Faculty of Engineering University of Jaffna and our families.

\section{References}

[1] National Transport Statistics 2015 National Transport commission of Sri Lanka

[2] https://en.wikipedia.org/wiki/Transport_in_Sri_Lanka

[3] ISO Standard of vibrations ISO2631-1,ISO2631-4 and ISO2631-5

[4] Transmission of vertical whole body vibration to the humanbody, journal of bone and mineral research volume 23, number 8, 2008 published online on march 17, 2008; doi: 10.1359/jbmr.080315 2008 american society for bone and mineral research

[5] Mayao Wang, Xing Gao, Adel Abdel-Wahab, Simin Li, Elizabeth A. Zimmermann, Christoph Riedel, Bjorn Busse, and Vadim V. Silberschmidt,Effect of micromorphology of cortical bone tissue on crack propagation under dynamic loading EPJ Web of Conferences 94, 03005 (2015) DOI: 10.1051/epjconf/20159403005 Owned by the authors, published by EDP Sciences, 2015

[6] Jens Trampe Broch,Mechanical Vibration and Shock Measurements by ISBN 87873553451984

[7] Rasmussen, G., Bruel and Kjaer,Human body vibration exposure and its measurement. 1982.

[8] Zhenhua Yan, Bing Zhu, Xuefei Li, and Guoqiang ,Modeling and Analysis of Static and Dynamic Characteristics of Nonlinear Seat Suspension for Off Road Vehicles Hindawi Shock and Vibration Volume 2015 (2015), Article ID 9382052015

[9] Ann M. Nakashima , The effect of vibration on human performance and health A review of recent literature Defence R\&D Canada Toronto Technical Report DRDC Toronto TR 2004-089 July 2004

[10] Min-Soo Park, Takabumi FUKUDA, Tae-gu KIM and Setsuo Maeda, Health Risk Evaluation of Whole-body Vibration by ISO 2631-5 and ISO 2631-1 for Operators of Agricultural Tractors and Recreational Vehicles Industrial Health 2013, 51, 364-370 April 5, 2013

[11] Manal El Sayed, Shahira Habashy, Mohamed El Adawy, Evaluation of Whole-Body-Vibration Exposure to Cairo Subway (Metro) Passengers International Journal of Computer Applications (0975 8887) Volume 55- No.8, October 2012

[12] Silviu Nastac, Mihaela Picuthe Evaluating methods of whole-bodyvibration exposure in trains annals of "dunarea de jos" university of galati fascicle xiv mechanical engineering,ISSN 1224-5615 2010

[13] Anil K. Chopra, Dynamics of Structures ISBN 13:978-0-13-285803-8 2013.

[14] Sakakibara H, et al. Digital nerve conduction velocity as a sensitive indication of peripheral neuropathy in vibration syndrome. Am J Indus Med 1994;26:359-366.

[15] Sakakibara H, et al. Affected segments of the median nerve detected by fractionated nerve conduction measurement in vibration-induced neuropathy. Indus Health 1998; 36:155-159.
[16] Jiu-Chiuan Chen, Wen-Ruey Chang, Britt H. Hatfield, David C Christiani characteristics of whole-body vibration frequencies and low back pain in urban taxi drivers Proceedings of the First American Conference on Human Vibration June 5-7, 2006 Morgantown, West Virginia, U.S.A.

[17] Dennis r. carter, william e. caler, dan m. spencler \& victor h. frank fatigue behavior of adult cortical bone:the influence of mean strain and strain range acta orthopaedica scandinavica November 1981

[18] Abbey M. Campbell, Michelle L. Cler, Carolyn P. Skurla, and Joseph J. Kuehl Damage accumulation of bovine bone under variable amplitude loads Elservier Bone Rep. 2016 Dec; 5: 320-332.

[19] M. P. Miller, D. L. McDowell and R. L. T. Oehmke A Creep-FatigueOxidation Microcrack Propagation Model for Thermomechanical Fatigue J. Eng. Mater. Technol 114(3), 282-288 (Jul 01, 1992).

[20] Jacob Rosen and Mircea Arcan Modeling the Human Body/Seat System in a Vibration Environment Journal of Biomechanical Engineering APRIL 2003, Vol. 125 Õ 223.

[21] C.E. Clauser, J.T. McConville, J.W. Young weight, volume, and center of mass of. segments of the human body Aerospace medical research laboratory and NASA August 1969.

[22] David A. Winter Biomechanics and Motor Control of Human Movement, Fourth Edition ISBN: 978047039818017 SEP 2009.

[23] Y. C. Fung Biomechanics: Mechanical Properties of Living Tissues Springer, Jun 18, 1993.

[24] C. Sujatha, P.V. Phaskara Rao, S. Narayana whole body vibration in Indian busses International journal of heavy vehicle systems 1995

R. Wijesiriwardana:

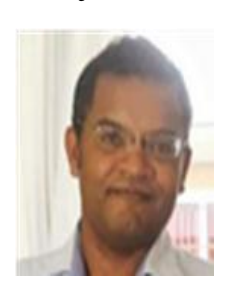

Dr. Ravi is specialized in the multidisciplinary field of wearable physiological information monitoring systems, RF and Acoustic Engineering. He received his Ph.D. from University of Manchester UK from the Faculty of Engineering and Physical Sciences in 2005.

M. Vignarjah:

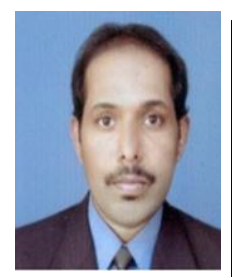

Eng. M. Vignarajah obtained his B.Sc.(Eng) degree with Second Class Honors from the University of Peradeniya in Year 2000. Also he earned M.Phil degree from University of Peradeniya in Year 2005.

P.Kathirgamanathan:

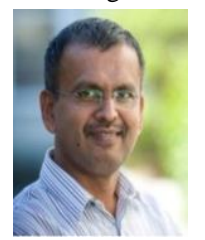

Dr Padmanathan Kathirgamanathan is a senior lecturer with a background in mathematics and engineering. He joined the Faculty in 2015. 